\title{
User Experience Research at Tech Mahindra
}

\author{
Sanjay Tripathi \\ User Interaction Design Group, Tech Mahindra Limited \\ Sharda Centre, Pune- 411005 India \\ stripathi@techmahindra.com
}

\begin{abstract}
In this overview we describe how user experience research and design has been established in Tech Mahindra Ltd. (TechM), and how it is organised to support both short term development programs and long term research. While focusing on research activities at TechM, the challenges of assessing real user experience issues critical to business success, user experience measurement methodology and future research focus are discussed in this paper.
\end{abstract}

Keywords: Social computing, user experience metrics, index of integration, Telecom, Interaction design, HCI.

\section{Introduction}

Tech Mahindra Limited (TechM) is the global leader in providing end-to-end IT services and solutions to the Telecom industry. Over 25,000 professionals service clients across various telecom segments, from multiple offshore development centres across cities in India, UK and sales offices across America, Europe and Asia-Pacific.

TechM recognised six years ago, the key role User Experience plays in ensuring the customer satisfaction for its clients' services and products, and started building the capability in the domain of User Experience Design and Usability Engineering.

Advancements in the technology that has led to proliferation of access devices, pervasive presence of Internet, and the changing lifestyle of users have increased the complexity of developing the User Experience solutions.

The Service Delivery at TechM therefore is based on the philosophy of integration of user centred approach, customers' business imperatives and software engineering practices. The Research in this domain is carried out to support this philosophy. Our Research Group is distributed across locations, based on the demands of universal usability and local cultural influences on diversified user base of TechM's clients.

\section{User Experience Design at Tech Mahindra}

The TechM's User Interaction Design Group (UIDG) provides Usability and User Experience Design services to global customers. The group has deep theoretical knowledge combined with vast hands-on exposure in end-to-end User Experience solutions. The unique combination of capabilities and the multidisciplinary background 
of UIDG enable us to formulate a comprehensive approach taking into account the key dimensions of design, usability, technology and management.

At TechM, we follow a time tested methodology - UESDM. User Experience Solution Design Methodology process is unique and has evolved over the years with exposure to multiple assignments. UESDM is an iterative and methodical approach. It takes into consideration end user needs, business goals and usability best practices to successfully deliver end-to-end User Experience solutions.

\section{Recent Success}

Our team of experts has improved the ease of use of over 100 applications by offering the end-to-end design solutions, understanding the varied end user needs, diverse domains, overcoming the business challenges and technology constraints.

Research and capability division of UIDG at TechM started exploring new possibility of redefining users experience and its relevance for the business customers. It was often asked by our customers to provide better insight of users while they use their sites. We found that reports from web analytics (WA) tools alone are not adequate to provide insights in User Experience aspects. We have been successful in extending the capability of WA tool while incorporating User Experience attributes to the clickstream data generated by them.

We have also been successful in developing new User Experience Metrics (UXM) and its Index of Integration (IOI) in a collaborative research with Indian Institute of Technology.

UXM is an empirical inspection based User Experience on a scale of 0-100. The purpose of this metrics is to predict the quality of User Experience of a product early in a quantitative manner and without the help of user-based evaluation methods. This proved as effective measurement technique which is capable to predict the 'quality' of User Experience of a product.

IOI is an empirical process metric that represents the best possible integration of HCI activities in the software development. IOI helps to evaluate the impact of various HCI design activities on User Experience quality of the resulting product in a given domain.

\section{Vision for Future}

The field of Human Computer Interaction design being multi disciplinary and research oriented, TechM's UIDG has strong focus on the research activities. Keeping this perspective in mind, UIDG has already started working in the areas of Web 2.0, Rich Internet Application (RIA) and Web Analytics as immediate focus areas. Mobile user experience, alternative interaction style, alternative interfaces, and integrated social interaction etc. are the vision for future where we are aimed to bring our experience and expertise. 